\title{
Afrikas künstliche Grenzen: Koloniale Erblast oder Gebot der Vernunft?
}

\author{
Von Heinz Schneppen
}

Die politische Landkarte des heutigen Afrika sieht, was die Grenzen betrifft, nicht sehr viel anders aus als die von 1945 oder 1919. Die kolonialen Grenzen Afrikas sind - mit wenigen Ausnahmen - auch die Grenzen der heutigen Staaten. Mit der Unabhängigkeit haben die Namen gewechselt; das koloniale Gehäuse der Grenzen ist geblieben. Aber der Ruf nach einer Revision dieser Grenzen ist nie verstummt, auch wenn er sich nicht mehr so stark wie vor der Unabhängigkeit äußert.

Am Vorabend der Unabhängigkeit hatte 1958 die All-African Peoples Conference in Accra die Beseitigung der "künstlichen Grenzen" gefordert, die von imperialistischen Mächten in Afrika gezogen worden seien, um die Völker Afrikas zu teilen, insbesondere jene, die ethnische Gruppen und Menschen gleicher Abstammung trennten. ${ }^{1}$ Ein Jahr später fordert Kwame Nkrumah, die führende afrikanische Stimme seiner Zeit, die Afrikaner auf, Wege zu finden, "um die künstlichen Grenzen und Teilungen auszulöschen, die für die Balkanisierung unseres Kontinents verantwortlich sind"2. Das Stichwort der Balkanisierung ist auch das Leitmotiv einer jüngeren Veröffentlichung des britischen Historikers Basil Davidson über den "Fluch des Nationalstaats", den er als die "Bürde des schwarzen Mannes" bezeichnet. ${ }^{3}$ Mit ihrer Entscheidung für das europäische Staatsmodell hätten die afrikanischen Staaten sich bei ihrer Unabhängigkeit für eine Lösung entschieden, die weder afrikanischer Tradition noch af rikanischen Bedürfnissen entspreche.

In dieser Sicht sind die territorialen Kontlikte Afrikas eine Konsequenz jener Grenzen, die willkürlich von Europäern gezogen und bewußt von Afrikanern übernommen worden seien. Die ethnischen Auseinandersetzungen am Horn von Afrika und im Sudan scheinen hierfür ebenso ein Beweis wie frühere Versuche einer Sezession in Katanga oder Biafra. Vor diesem Hintergrund hat der Präsident von Ruanda, Pasteur Bizimungu, eine Revision der afrikanischen Grenzen und die "Berlin II-Konferenz" zur Korrektur der Berliner Konferenz von 1884-85 gefordert. Er hat dabei einem verbreiteten Gefühl Ausdruck gegeben, daß die

Touval, Saadia, The Boundary Politics of Independent Africa, Cambridge, Mass., 1972, p. 57.

2

Shaw, Malcolm, Title to Territory in Africa, International Legal Issues, Oxford, 1986, p. 183.

3 Davidson, Basil, The Black Man's Burden. Africa and the curse of the Nation-State, Oxford, 1992. 
Beschlüsse der Organisation für afrikanische Einheit (OAU) von 1963 und 1964 über die "Respektierung der kolonialen Grenzen" jene Teilung Afrikas verewigt haben, für die die Berliner Konferenz von 1884-85 zum Symbol geworden ist.

\section{Die Teilung Afrikas}

Dabei wurden auf der Berliner Konferenz zwar Verfahrensregeln für die Landnahme an der Küste bestimmt, aber keine Grenzen gezogen. Ziel der Konferenz war es vielmehr, die Prinzipien der Freiheit des Handels und der Schiffahrt auf das vertragliche "Kongobecken" zu erstrecken, das weit über den Kongostaat des Königs der Belgier hinausging. Das Ergebnis der Konferenz, die Kongo-Akte, war in den Worten Carl Schmitts "ein merkwürdiges letztes Dokument des ungebrochenen Glaubens an Zivilisation, Fortschritt und Freihandel und des auf diesen Glauben sich gründenden europäischen Anspruchs auf den freien, d.h. europäischer Okkupation offenstehenden Boden des afrikanischen Kontinents" ${ }^{4}$. Aber da die Konferenzteilnehmer das Erfordernis der "effektiven Okkupation" für die Landnahme in Afrika auf die Küste beschränkten und die Frage nach den Regeln für die Landnahme im Inneren offen ließen, haben sie, ohne es zu wollen, das Tempo der Teilung Afrikas beschleunigt. Sie haben sich dabei jener politischen und rechtlichen Mittel bedient, die ihren Interessen entsprachen und von ihren Konkurrenten akzeptiert oder toleriert wurden. Es handelte sich dabei um ein buntes Gemisch völkerrechtlicher Ansprüche, die sich auf Entdeckungen und Forschungen, symbolische und faktische, zumeist aber wenig effektive Besitzergreifungen sowie auf eine Vielzahl oft fragwürdiger Verträge mit einheimischen Häuptlingen stützten, denen die Konsequenzen ihres Handels nicht immer bewußt waren. "Der Erwerb von Land ist in Ostafrika sehr leicht", so wird Bismarck 1886 zitiert, "für ein paar Flinten besorgt man sich ein Papier mit einigen Negerkreuzen" lere Rechtstitel oder sonstige Anknüpfungspunkte fehlten, berief man sich auf Faktum oder Fiktion einer terra nullius, herrenloses Land. Mit ihrer Hinterland-Doktrin suchten sich 1890 die Deutschen in Ostafrika jene Gebiete westlich des Küstensaums zu sichern, den die Deutsch-Ostafrikanische Gesellschaft vom Sultan von Sansibar gepachtet hatte. Um ihren Traum einer ununterbrochenen Landverbindung "vom Kap nach Kairo" zu verwirklichen, beriefen sich die Briten nördlich des Tanganyikasees auf die Reisen Stanleys, südlich des Sees auf die Anwesenheit schottischer Missionare. Die Institution des "Protektorats" erwies sich als bequemes Mittel, äußere Souveränität über ein Staatswesen zu erlangen, dem man zusagte, seine innere Souveränität zu respektieren. Nach wenigen Jahren waren Protektorate wie Tunesien (1881), Madagaskar (1885), Sansibar (1890), Uganda (1895), Ägypten 
(1889) und Marokko (1912) von kolonialen Besitzungen schwer zu unterscheiden. ${ }^{6}$ Briten wie Deutsche bedienten sich gleichermaßen des Begriffs der "Einflußsphäre", den die Artikel 6 und 9 der Generalakte der Berliner Konferenz eingeführt hatten. Er eignete sich vorzüglich, territoriale Ansprüche dort geltend zu machen, wo die Kräfte noch nicht ausreichten, den Anspruch mit Souveränität zu füllen. Es war eine Option auf eine spätere Besitzergreifung, eine Abgrenzung zwischen zwei Partnern, die Dritte ausschlossen. Von denen, die das Land bewohnten und deren Land von den Grenzen durchschnitten wurde, war kaum die Rede. Als bei der Berliner Konferenz der amerikanische Delegierte die Frage aufwarf, ob nicht das Einverständnis derer, deren Land in Besitz genommen werde, von Bedeutung sei, stieß er auf keine Resonanz. Man zeigte keine Neigung, diese Frage zu vertiefen. Für "Wilde" außerhalb des westlichen Kulturkreises war das Völkerrecht nicht gedacht. Und das Selbstbestimmungsrecht der Völker war, als Afrika geteilt wurde, weder politische Praxis noch rechtliche Norm.

\section{Die kolonialen Grenzen in af rikanischer Perspektive}

In den zwanzig Jahren zwischen 1880 und 1900 haben die kolonialen Grenzen das Gesicht Afrikas grundlegend verändert. Aber die Abgrenzung ihrer Interessen in Afrika stellte auch die Diplomatie der europäischen Mächte vor neue Aufgaben. "Wenn Afrika, dieses dunkle Land, nicht existieren würde, hätten wir Diplomaten wenig zu tun", so Graf Münster, kaiserlicher Botschafter in London, 1890 in einem Brief an den Bankier Bleichröder, den finanzielle Interessen mit dem Kongoprojekt des Königs der Belgier verbanden. ${ }^{7}$ Der britische Premierminister Lord Salisbury hat im gleichen Jahr nicht verschwiegen, auf welch unzureichenden geographischen Kenntnissen die Entscheidungen der europäischen Kabinette beruhten: "Wir waren damit beschäftigt, auf Landkarten Linien dort einzuzeichnen, wo noch nie ein Weißer seinen Fuß gesetzt hatte; wir haben einander Berge, Flüsse und Seen abgetreten, die nur den Nachteil hatten, daß wir nie genau wußten, wo diese Berge, Flüsse und Seen eigentlich waren." ${ }^{8}$ Dies galt auch für den Fall des Mt. Mfumbiro, den die Deutschen den Briten im Helgoland-Sansibar-Vertrag von 1890 konzedierten, der sich aber auch nach 10jährigem Suchen als unauffindbar erwies. Da er den Briten - so eine Lesart als Ausgleich für den Verlust des Kilimandscharo zugedacht war, fiel es den Briten schwer, auf den Berg zu verzichten, den Stanley bei seinen Forschungsreisen im Dreieck von Kongo, Ruanda und Uganda gesichtet haben wollte. Erst im Kivu-Vertrag von 1910 einigten sich Deutsche und Briten, weitgehend zu Lasten der Belgier, die Kette der

6

7

8

Shaw (oben Fn. 2).

Stern, Fritz, Gold and Iron. Bismarck, Bleichröder and the Building of the German Empire, New York, 1979, p. 411.

Hargreaves, J.D., The Making of the Boundaries, in: A.I. Asiwaju, Partitioned Africans, London, 1985 , p. 22. 
Virunga-Vulkane als Grenze ihrer Territorien und als Kompensation für den nicht zu identifizierenden Mt. Mfumbiro zu betrachten.

Mangelnde Kenntnisse der Geographie haben daher in vielen Fällen dazu geführt, sich beim Prozeß der Abgrenzung astronomischer oder geometrischer Linien zu bedienen. Die so festgelegten Grenzen waren abstrakt, aber eindeutig. Daß sie, wie ein Messer, lebendes Gewebe durchschnitten, war weniger für die Chirurgen als für die Patienten von Bedeutung. Über 187 "Stammesterritorien" hat K.M. Barbour 1961 gezählt, die im tropischen Afrika von kolonialen Grenzen geteilt würden. ${ }^{9}$ Über 100 Ethnien enthält die Liste des nigerianischen Historikers Asiwaju ${ }^{10}$, die durch die (1985) 103 internationalen Grenzen des afrikanischen Kontinents getrennt würden. Mit 53 Staaten ist Afrika der staatenreichste Kontinent der Erde überhaupt. Entsprechend zahlreich sind seine Grenzen. Entsprechend groß ist das Potential für Konflikte.

Als die Europäer Afrika teilten, fühlten sich die Afrikaner von den kolonialen Grenzen zunächst nicht betroffen. "Für uns ist die Grenze etwas, was die Briten und die Franzosen teilt, nicht die Yoruba", so der Alaketu (König) von Ketu im heutigen Benin ${ }^{11}$. So mögen zunächst auch die Massai gedacht haben, als Briten und Deutsche 1886 wie mit dem Lineal ihre Einflußzonen zwischen dem Indischen Ozean und dem Victoria-See voneinander trennten, wobei die gerade Linie nur durch den Kilimandscharo unterbrochen wurde, den sich die Deutschen gegen die Briten sicherten, denen sie dafür Mombassa überließen. ${ }^{12}$ Es kann daher nicht überraschen, daß die Massai 1898 die deutsch-britische Grenze nördlich des Kilimandscharo ignorierten, wo Sir Arthur Hardinge die britische Regierung bei den Vermessungsarbeiten vertrat: "Unser Massaifreund unterrichtete uns, daß wir uns in einem Irrtum befänden, da das Land nicht den Europäern, sondern seinem Stamme gehöre. Sollte dies strittig sein, müsse der Streit zwischen Lenana und Sendeyo, den Erben des großen Medizinmannes M'batian geklärt werden. Da es sinnlos war, sich gegenüber einem primitiven Wilden auf europäische Verträge zu beziehen, haben wir ihm nur erklärt, daß, sofern in Zukunft sich irgendwelche Fragen hinsichtlich der Rechte seiner Leute ergäben, sie sowohl uns wie den Deutschen vorgetragen werden müßten" ${ }^{13}$. Ein Vierteljahrhundert später

Barbour, K.M., A Geographical Analysis of Boundaries in Inter-tropical Africa, in: K.M. Barbour/ R.M. Prothero, Essays on African Populations, London, 1961, pp. 312-313.

10

Asiwaju, A.I., Partitioned Culture Areas: A Checklist, in: A.I. Asiwaju, Partitioned Africans, London, 1985, pp. 252-259.

11 Asiwaju, A.I., The Conceptual Framework, in: A.I. Asiwaju, Partitioned Africans, London, 1985, p. 9.

12

Hier und zur Legende über das angebliche Geschenk von Queen Victoria an ihren Enkel Wilhelm II. siehe Schneppen, Heinz, Why Kilimanjaro is in Tanzania, Occasional Paper No. 9, National 13 Museum of Tanzania, Dar es Salaam, 1996.

Hardinge, Sir Arthur, A Diplomatist in the East, London, 1928, p. 232. 
beschäftigte sich der Völkerbund mit der Lage der halbnomadischen Massai, deren Lebensraum nun von den Grenzen durchschnitten wurde, die die britische Kronkolonie Kenia von dem (jetzt) britischen Mandatsgebiet Tanganyika trennten. Aber alle Bemühungen, das geteilte Territorium der Massai ungeteilt entweder an Kenia oder Tanganyika anzuschließen, blieben ohne Erfolg. Auch als beide Länder unabhängig wurden, gab es dafür keine Chance.

Aber der Fall der Massai ist nur einer von denen, die unser Länderbeispiel Tansania berühren. Im Süden des Landes lebten die Makonde, die 1886 die koloniale Grenzziehung entlang dem Ruvuma von ihren Stammesgefährten in Mozambik trennte. Der Stamm der Haya erstreckte sich vom nordwestlichen Tansania ins südliche Uganda, was Idi Amin 1979 als Anlaß diente, tansanisches Territorium südlich des $1^{\circ}$ Grades südlicher Breite zu besetzen, der seit 1890 die Grenze zwischen dem britischen Protektorat Uganda und dem deutschen Schutzgebiet bildete. Zwischen dem Tanganyika- und dem Nyassasee wurden einige kleinere Stämme von der kolonialen Grenze des deutsch-britischen Vertrages von 1890 geteilt. Derselbe Vertrag trennte aber auch die Insel Sansibar von der Suaheli-Bevölkerung der Festlandsküste, mit der sie politisch, wirtschaftlich und kulturell eng verbunden war. Für Julius Nyerere bedeutete daher die Union von Sansibar und Tanganyika 1964 die Wiederherstellung einer alten Einheit, die durch einen "Unfall der Geschichte" - den HelgolandSansibar-Vertrag - verlorengegangen war, als die Deutschen das Festland als "Schutzgebiet", die Briten die Insel als "Protektorat" in Besitz nahmen.

Aber im afrikanischen Vergleich ist Tansania ein wenig problematischer Fall. Tansanias Grenzen werden heute weder von außen bedroht noch von innen in Frage gestellt. Benachteiligt ist Tansania aufgrund der kolonialen Grenzziehung jedoch am Nyassasee. Er wurde so geteilt, daß Malawi und Mozambik unter Berücksichtigung der Mittellinie die Souveränität über die Seefläche ausüben, während Tansania durch die Verträge von 1890 und 1954 der Zugang zum See verweigert wird. Während dieses Problem seit Jahren "schläft", hat in anderen Ländern Afrikas die koloniale Grenzziehung Spannungen oder Konflikte erzeugt, von denen viele latent, andere jedoch virulent sind. Aber selbst dort, wo die Grenzen nicht problematisch sind, erschweren sie Kontakt und Kommunikation zwischen geographischen oder ethnischen Nachbarn. Dies gilt nicht zuletzt für das Verhältnis zwischen den frankophonen und anglophonen Ländern des Kontinents, wo sich die territorialen Grenzen mit einer kolonial bedingten Sprachbarriere verbinden.

Überlagern wir die Stammeskarte Afrikas mit der Karte der politischen Grenzen, fallen die Gebiete ins Auge, wo sich "Staat" und "Stamm" überschneiden, aber nicht decken. Die Ewe leben in Togo wie in Ghana, Haussa und Fulani in Nigeria und in Niger. Die Bakongo siedeln auf einem Gebiet, das staatlich Gabun, Angola und den beiden Kongo-Republiken zugeordnet ist. Lunda gibt es in Angola, Sambia und der Demokratischen Republik Kongo (Zaire). Die Zande sind durch die Grenzen auf den Sudan, Tschad, die Zentralafrikanische 
Republik und die Volksrepublik Kongo verteilt. Yoruba und Aja finden sich getrennt in Nigeria, Benin und Togo. Die Gourma bewohnen Teile von Burkina Faso, Togo und Benin. Wolof und Serers leben gleichermaßen in Gambia und Senegal. Soninke und Tukulor sind durch die Grenze zwischen Senegal und Mauretanien geteilt. Die Grenze zwischen Tschad und Libyen wie die zwischen Tschad und Niger trennt Tuareg und Tubu. Nubier finden sich auf beiden Seiten der Grenze zwischen Ägypten und dem Sudan. Die Tswana werden von der Grenze zwischen Botswana und Südafrika auseinandergerissen. Die Herero weiden ihre Schafe, die "Buschmänner" suchen ihr Wild auf beiden Seiten der Grenze zwischen Namibia und Botswana. ${ }^{14}$ In einer Reihe von Fällen wird die ursprüngliche koloniale Grenzziehung durch spätere Grenzveränderungen weiter kompliziert. Vor allem die französische Kolonialmacht hat die inneren administrativen Grenzen ihres westafrikanischen Besitzes immer wieder verschoben. So haben die Grenzen Malis zwischen 1890 und 1947 verschiedentlich gewechselt, wobei Teile Malis dem Senegal, Sudan, Niger und Obervolta zugeschlagen wurden. Obervolta, 1919 konstituiert, wurde 1932 wieder liquidiert, wobei sein Gebiet auf den Sudan, Niger und die Elfenbeinküste verteilt wurde. 1932 wurde Obervolta aus seinen früheren Elementen neu errichtet, wobei dieser Maßnahme politische, nicht ethnische Überlegungen zugrunde lagen. ${ }^{15} 1902$ wurde die östliche Provinz Ugandas an das britische Protektorat Ostafrika angeschlossen, um den schon fertiggestellten Teil der Uganda Railway einer einzigen Verwaltung zu unterstellen. ${ }^{16}$ Als Folge des Ersten Weltkriegs wurde das deutsche Schutzgebiet Togo zwischen Briten und Franzosen aufgeteilt. Der Vertrag von Versailles war ebenfalls die Grundlage für die Teilung des früher deutschen Kamerun zwischen Großbritannien und Frankreich, denen 1922 die geteilten Gebiete als Mandat des Völkerbundes übertragen wurden. ${ }^{17}$

Damit ist die Liste keineswegs komplett. Auch ist das Konfliktmuster nicht immer das gleiche. Im östlichen Nigeria sah der Stamm (oder die Nation?) der Ibo keinen Ausweg als die Sezession. Im Sudan befinden sich Nord und Süd seit fast einem halben Jahrhundert im Bürgerkrieg, der sich nicht auf eine einzige Ursache reduzieren läßt. Noch komplexer ist die Lage in Somalia, wo sich der einzige homogene Nationalstaat Afrikas im Staatszerfall befindet. Unterschiedlich wie die Fälle sind - für alle sind die kolonialen Grenzen von Bedeutung.

14

15

16

17

Brownlie, Ian, African Boundaries. A legal and diplomatic Encyclopedia, London, 1979, pp. 533- 


\section{Die kolonialen Grenzen in der Sicht Europas}

Im Prozeß gegen die Kolonialmächte von einst scheint die Anklage über erdrückende Beweise zu verfügen. Aber auch das Urteil der Geschichte kann nicht umhin, jene Gründe zu berücksichtigen, die geeignet sein könnten, die Täter von einst zu entlasten. Die Verteidigung und ihre Zeugen werden sich dabei auf die Mitwirkung der Afrikaner berufen. Sie werden die verbotene Rückwirkung von Normen und Regeln geltend machen, die damals noch nicht galten. Sie werden auf den Wissensstand der Zeit und die fehlenden geographischen und ethnographischen Kenntnisse verweisen. Sie werden die positiven Wirkungen ihres Handelns unterstreichen oder die Frage nach der Alternative stellen. Sie werden zwar nicht auf Freispruch plädieren, aber auf mildernde Umstände.

Die Aufteilung Afrikas stand als "letzte gemeinsame Landnahme nicht-europäischen Bodens durch europäische Mächte" ${ }^{18}$ in der Tradition europäischer kolonialer Expansion. Sie hatte mit der gewaltsamen Unterwerfung der beiden Amerikas begonnen, um sich später nach Asien fortzusetzen, wo es nur wenigen Ländern gelang, ihre Unabhängigkeit zu erhalten. Nach den Forschungsreisen von Livingstone, Stanley, Burton, Speke und de Brazza schien Afrika reif für Ausbeutung und Entwicklung. Wenn die Europäer Afrika in Besitz nahmen und ihren Besitz gegeneinander abgrenzten, hatten sie dabei kein schlechtes Gewissen, ging es doch darum, Afrika "Civilisation and Commerce" zu öffnen, wie es die führende Kolonialmacht der Zeit formulierte. Die Verbreitung des Christentums und die Abschaffung der Sklaverei waren zusätzliche Motive. So verbanden sich auf der Berliner Kongo-Konferenz materielle Interessen mit ideellen Zielen. Großbritannien, Frankreich und die Niederlande hatten sich bereits in Afrika etabliert. Jetzt drängten Deutschland und Italien als "verspätete Nationen" nach vorn. Aber auch der König der Belgier wollte seinen Teil des Kuchens.

Dieser Kuchen war groß, waren 1880 doch erst rund $18 \%$ des afrikanischen Kontinents europäischer Herrschaft unterworfen. ${ }^{19}$ Davon waren $70 \%$ Gebiete an der Küste. Noch befand sich das Hinterland weitgehend außerhalb europäischen Einflusses. Diese Gebiete aber waren meist so dünn besiedelt, daß die spärliche europäische Präsenz kaum wahrgenommen wurde. Die abstrakten Grenzen, die die Europäer damals zogen, schienen späteren Generationen "künstliche" Linien auf Papier, die man kritisch mit den "natürlichen" Grenzen Europas verglich. Aber: "Die Natur kennt keine Grenzen als die, die ihr der Mensch auferlegt", so der ägyptische Völkerrechtler und spätere Generalsekretär der Vereinten Nationen Boutros-Ghali. ${ }^{20}$ Weder sind Grenzen, die sich an "natürlichen" Gegebenheiten

Schmitt (oben Fn. 4), S. 188.

Hangula, Lazarus, Die Grenzziehung in den afrikanischen Kolonien Englands, Deutschlands und 20 Portugals im Zeitalter des Imperialismus 1880-1914, Frankfurt am Main, 1991, S. 24.

Boutros-Ghali, Boutros, Les Conflits de Frontières en Afrique, Paris, 1973, p. 11. 
orientieren, unbedingt besser als solche, die mit dem Lineal am Kartentisch gezogen wurden, noch ist die Grenzziehung unter Druck ein afrikanisches Phänomen. Die Tatsache, daß in Europa viele Grenzen Kriegsfolgen sind, spricht nicht für die Friedlichkeit und Freiwilligkeit dieser territorialen Veränderungen. Was die Grenzen in Afrika von denen in Europa unterscheidet, ist der zeitliche Faktor. Wozu man in Europa Jahrhunderte brauchte, vollzog sich in Afrika in wenigen Jahrzehnten. Europas Grenzen waren in Jahrhunderten entstanden, Afrikas Grenzen wurden in Jahrzehnten geschaffen. In Europa waren die Grenzen das Ergebnis eines historischen Prozesses, in Afrika dessen Beginn.

Es ist nicht zu bestreiten, daß die kolonialen Grenzen in einer Vielzahl von Fällen gewachsene, ethnische, kulturelle und linguistische Einheiten zersplitterten. Andere Grenzen, wie die des Caprivi-Zipfels, erwecken den Eindruck von Willkür, auch wenn diesem - über 400 Kilometer langen und nur wenige Kilometer breiten - Konstrukt die Absicht zugrunde lag, Deutsch-Südwestafrika mit dem Sambesi zu verbinden. Richtig ist aber auch, daß die Kolonialmächte nicht generell ethnische Gesichtspunkte ignorierten. Sie haben in ihren Verträgen durchaus Flexibilität bewiesen und dem Gesichtspunkt der Integrität von Stammesgebiet in einer Reihe von Fällen Rechnung getragen. So bestimmte der anglo-französische Vertrag von 1890, daß die Grenze zwischen den späteren Staaten Nigeria und Niger in einer Weise gezogen werden sollte, die Niger das Gebiet zuschlug, "das zu Recht dem Königreich Sokoto" zuzurechnen sei. Ein Vertrag zwischen Großbritannien und Portugal von 1891 legte fest, daß die Abgrenzung ihrer zentralafrikanischen Territorien entlang der Westgrenze des Königreichs Barotse verlaufe, um dessen Gebiet nicht zu gefährden. ${ }^{21}$ Ian Brownlie's umfassende Bestandsaufnahme der afrikanischen Grenzen ${ }^{22}$ enthält zahlreiche Grenzabschnitte, bei deren Festlegung die Stammesgrenzen Berücksichtigung fanden. Aber die Kolonial- bzw. Mandatsmächte waren in einigen Fällen auch nachträglich bereit, die Grenzen ihrer Territorien zu ändern, wo sie offenkundig nicht den Interessen der einheimischen Bevölkerung entsprachen. So wurde 1923 ein Gebiet von rund 5000 Quadratkilometern westlich des Kagera wieder mit Ruanda vereinigt, das erst 1919 dem britischen Mandatsgebiet Tanganyika zugeschlagen worden war. Das Gebiet sollte den Briten den Bau einer Eisenbahnlinie von Tanganyika nach Uganda erleichtern, wobei aber of fenbar niemand den Auswirkungen auf die Bevölkerung Beachtung schenkte. Der ruandische König Musinga wies drastisch darauf hin, daß man mit dieser Grenze sozusagen eines seiner Beine amputiere. Er fand bei seinem Protest die entschiedene Unterstützung von Missionaren beider christlicher Konfessionen, so daß der Völkerbund den alten Zustand wieder herstellte. $^{23}$ Fn. 1), p. 9. 
Aber auch dort, wo die Grenzen nicht zur Disposition standen, sind die mit der späteren Vermessung und Markierung beauftragten Gemischten Kommissionen von ihren Regierungen ermächtigt worden, ad hoc in begrenztem Umfang von der vereinbarten Grenzlinie abzuweichen, wenn dies die Rücksicht auf die Bedürfnisse der einheimischen Bevölkerung erfordere. Auch enthielten Abgrenzungsverträge Bestimmungen zugunsten der Grenzbevölkerung. So sicherte der (nicht mehr ratifizierte) deutsch-britische Vertragsentwurf von 1914 an der heutigen Grenze von Kenia und Tansania den Bewohnern die Nutzung von Wasserstellen jenseits der Grenze zu. ${ }^{24}$ Durch den Vertrag zwischen Äthiopien und Britisch-Somaliland von 1897 wurde den halbnomadischen Viehzüchtern und ihren Herden der Grenzübertritt erleichtert und der Zugang zu traditionellen Weidegründen jenseits der Grenzen eingeräumt. $^{25}$

Diplomaten und Kolonialbeamten war durchaus bewußt, daß es in ihrem Interesse lag, vorgegebene politische und soziale Strukturen zu nutzen. Indem sie sich im System einer "indirect rule" mit den Grenzen der einheimischen Herrscher identifizierten, erweiterten und stabilisierten sie ihre eigene Herrschaft. War dies besonders bei den Briten ausgeprägt, so galt dies zum Teil auch für die Deutschen. Als Leopold von Belgien sich nach 1890 daran machte, die nur ungefähr bestimmte östliche Grenze des Kongostaates weiter nach Osten zu verschieben, stieß er auf den Widerstand der Deutschen, die aufgrund des deutsch-britischen Vertrages von 1890 das Gebiet des heutigen Ruanda und Burundi beanspruchten. Aber Leopolds "schräge Linie" hätte nicht nur die deutschen Ansprüche beschnitten, sondern auch die feudalen Reiche Ruanda und Burundi geteilt. Demgegenüber sah die deutsche Regierung in einer Linie am Rusisi zwischen Kivu- und Tanganyika-See eine ethnisch klare Grenze, die die Stämme Ruanda-Burundis von ihren westlichen Nachbarn trennte. Erst der Kivu-Vertrag von 1910 hat diese Grenze bestätigt, wobei sich die Deutschen ihrer eher schwachen juristischen Position durchaus bewußt waren. Intern hat die deutsche Regierung ihre Haltung mit der Notwendigkeit begründet, die territoriale Integrität Deutsch-Ostafrikas zu wahren, von dem Ruanda-Burundi der wichtigste Teil sei. Es sei notwendig, so argumentierte die deutsche Kolonialverwaltung in Daressalam, eine Auflösung des "Sultanats" Ruanda zu verhindern, da dies für das deutsche Schutzgebiet insgesamt negative Auswirkungen habe und zugleich die Sicherheit seines exponierten nordwestlichen Teils gefährde. ${ }^{26}$ Es konnte somit sehr wohl den Interessen der Kolonialmacht entsprechen, ihre eigenen Ziele ethnisch zu begründen, auch im Hinblick darauf, daß eine "horizontale" Teilung des Gebiets die "vertikale" Teilung der Bevölkerung nach Hutu und Tutsi weiter kompliziert hätte. Dies ändert jedoch nichts an der Tatsache, daß die einheimische Bevölkerung die Dispositionsmasse der Kolonialmächte darstellte, über die man

McEwen (oben Fn. 23), p. 40.

Brownlie (oben Fn. 17), p. 1351; Kapil, Ravil, On the Conflict Potential of inherited Boundaries in Africa, World Politics, Princeton, XVIII, 1965/66, p. 662.

26

Bundesarchiv, Reichskolonialamt, 594, 629, 630, 633, 636. 
der politischen Konstellation entsprechend verfügte. So wurde 1919, als das Deutsche Reich seine Kolonien verlor, Ruanda-Burundi den Belgiern als Mandat des Völkerbundes übertragen. Dabei war den Belgiern als Ausgleich für ihren Kriegsbeitrag sehr viel mehr daran gelegen, ihren Besitz an der Kongo-Mündung zu erweitern, wo sich der Kongostaat am Atlantik auf wenige Kilometer verengt. Die Briten waren ihrerseits daran interessiert, daß ihnen außer "Tanganyika" auch Ruanda und Burundi als geschlossenes Mandatsgebiet zugesprochen würden. Aber britische Bemühungen, die Portugiesen durch Territorium im Süden von "Deutsch-Ostafrika" (nördlich des heutigen Mosambik) zum Verzicht auf portugiesisches (heute angolanisches) Gebiet südlich der Kongo-Mündung zu bewegen, schlugen fehl. Ruanda und Burundi, bis dahin ethnisch, kulturell und kommerziell eher nach Osten orientiert, sahen sich nun faktisch mit der Kongokolonie verbunden, die sie als eher wesensfremd empfanden. Julius Nyerere, Präsident Tansanias von der Unabhängigkeit bis 1985 und eine respektierte internationale Persönlichkeit, hat in der Entscheidung von 1919 die Ursache einer unheilvollen Entwicklung gesehen, die noch nicht an ihr Ende gekommen scheint. Für ihn - und seine Landsleute - steht fest, daß die Geschichte einen anderen Verlauf genommen hätte, wenn "Deutsch-Ostafrika" und ein diesem entsprechendes britisches Mandat den kolonialen Zustand "als Einheit" verlassen und den Zustand der Unabhängigkeit geschlossen erreicht hätte. Nur in einem größeren territorialen, sozialen und wirtschaftlichen Rahmen hätten sich jene Gegensätze überwinden oder entschärfen lassen, die weniger auf ethnischen Gegensätzen als auf einer polarisierten Klassenstruktur beruhen, die sich auf engem Raum schließlich in einem Genozid entluden. In diesem Falle stellt sich die Frage, ob es nicht besser gewesen wäre, vorkoloniale feudale Strukturen und Grenzen zu beseitigen, als sie zu konservieren.

Die Frage nach den Wirkungen der kolonialen Grenzen läßt sich nicht beantworten, wenn man nicht zugleich die Frage stellt, was diese Grenzen teilten. Unser Bild des präkolonialen Afrika wird dabei von der Vorstellung geschlossener Stämme auf eigenem Territorium geprägt, in denen wir die Frühformen späterer Staaten erblicken. Die afrikanische Realität wird aber nicht zuletzt von Migrationen bestimmt, in denen wandernde Stämme seßhafte verdrängten oder die Leere des Raumes füllten. So stießen Niloten nach Süden vor, um im Gebiet der Großen Seen als Tutsi zu siedeln; Zulu drängten von Süden, um sich als Wagoni am Nyassasee niederzulassen. Politisch waren die Stämme mehr durch ein personales Loyalitätsverhältnis zum "Herrscher" als durch ein klar umrissenes Stammesgebiet definiert. Wo die Loyalität der Untertanen endete, endete auch die Herrschaft des Herrschers. Nach heutiger Kenntnis kommt wohl den "Stämmen" nicht immer jene Bedeutung zu, die man ihnen bisher beigemessen hat.

John Iliffe, einer der besten Kenner der afrikanischen Geschichte, hat am Beispiel Tansanias die Vielfalt eines Landes beschrieben, das zu Beginn des 19. Jahrhunderts nicht von identifizierbaren Stämmen bewohnt wurde, sondern von einer Vielzahl von Ethnien ohne sozialen Verbund, aber auch ohne innere Grenzen. Intensiver Ackerbau erforderte frühe 
Formen staatlicher Organisation; extensive Viehzucht machte sie entbehrlich.; für Nomaden war sie ohne Bedeutung. Stammesnamen bezeichneten nicht immer ethnische Identität, sondern oft nur die Region räumlicher Herkunft. Bildeten für viele Familien Sippe und Dorf weiterhin den Lebensraum, so entdeckten andere später den Vorteil der größeren Gemeinschaft, als deren Mitglieder sie sich den Europäern präsentierten: "Europeans believed Africans belonged to tribes; Africans built tribes to belong to" 27 .

Auch wenn diese Analyse nicht für alle Teile Afrikas gilt, so spricht doch vieles dafür, den "Stamm" nicht als ein ethnisches Urphänomen, sondern als ein soziales Gebilde zu betrachten, das sich in Afrika in unterschiedlichen Formen und unterschiedlicher Verdichtung präsentierte und sich nicht nur auf das "Ethnos" der Abstammung, sondern auch auf das "Ethos" der Zuordnung gründete. Wird so der "Stamm" ein wenig entmythologisiert, so relativiert sich damit auch der pauschale Vorwurf an die europäische Adresse, durch willkürliche oder künstliche Grenzen eine ursprüngliche afrikanische Stammeslandschaft zerstört zu haben, die sich manchem Betrachter als gewaltfreier menschlicher Urzustand darstellt. Die Realität ist komplexer. Die Frage nach dem Stellenwert der Stämme ist aber nicht nur für die Bewertung kolonialer Grenzen, sondern auch für die Lebensfähigkeit der nachkolonialen Staaten von Bedeutung.

\section{Die Respektierung der kolonialen Grenzen: ein Gebot der Vernunft}

Als sich im Mai 1963 die unabhängigen afrikanischen Staaten in Addis Abeba versammelten, war die Gründung der "Organisation of African Unity" (OAU) das zentrale Thema der Konferenz. Aber die Frage der Grenzen forderte ebenso die Aufmerksamkeit der Teilnehmer, zumal die Ausarbeitung der Charta der Organisation eine grundsätzliche Stellungnahme ohnehin erforderlich machte. Die Erinnerung an die Wirren im Kongo und die Sezession Katangas war noch frisch. Spannungen zwischen Ghana und Obervolta, Ghana und Togo hatten zur Schließung dieser Grenzen geführt. Ein Konflikt zwischen Algerien und Marokko zeichnete sich ab, auch wenn Marokko erst einige Monate später algerisches Grenzgebiet besetzte. König Hassan von Marokko war der Konferenz aus Protest gegen die Teilnahme Mauretaniens ferngeblieben, das er als Teil Marokkos betrachtete. Im Namen der Einheit der Nation erhob Somalia territoriale Forderungen gegen Äthiopien und das noch abhängige Kenia. Aber auch andere afrikanische Staaten hatten mit den kolonialen Grenzen ihre Probleme.

Hatten viele afrikanische Führer vor der Unabhängigkeit eine Revision ihrer Grenzen gefordert, so veränderte die Unabhängigkeit ihre Perspektive. Für den Präsidenten von Mali, Modibo Keita, stand fest, "daß wir Afrika so nehmen müssen, wie es ist. Wir müssen 
auf alle territorialen Ansprüche verzichten, wenn wir nicht eine Art schwarzen Imperialismus in Afrika einführen wollen... Die Einheit Afrikas fordert von jedem von uns völligen Respekt vor dem Erbe unseres kolonialen Systems; das heißt: Erhaltung der gegenwärtigen Grenzen unserer jeweiligen Staaten"28. Noch pointierter äußerte sich der Präsident von Madagaskar, Tsiranana: "Es ist nicht länger möglich und auch nicht wünschenswert, unter dem Vorwand rassischer, religiöser oder linguistischer Kriterien die Grenzen der Nationen zu ändern... Würden wir diese Kriterien benutzen, um unsere Grenzen festzulegen, so würden eine Reihe von Staaten in Afrika von der Karte verschwinden" ${ }^{29}$. Gegen den Widerstand Marokkos und Somalias hat diese Mehrheitsmeinung it der Charti inren Niederschlag gefunden. Die Präambel bekräftigt "die Souveränität und territoriale Integrität" der Mitgliedstaaten. Nach Artikel 2 und 3 sind Souveränität, territoriale Integrität und Unabhängigkeit Ziele und Prinzipien der Organisation. Artikel 19 verpflichtet alle Mitgliedstaaten, ihre Streitigkeiten auf friedlichem Wege beizulegen. Hierfür steht den Milgliedstaaten eine Kommission für "Mediation, Conciliation und Arbitration" zur Verfügung.

Als die zweite Gipfelkonferenz der OAU im Juli 1964 in Kairo zusammentrat, war die Lösung der Grenzfragen noch dringender geworden. Die OAU war aufgefordert, im Krieg zwischen Marokko und Algerien zu vermitteln und zur Lösung des Konflikts beizutragen, der sich für die Nachbarn aus dem somalischen "Irredentismus" ergab. Streit zwischen Ghana und Obervolta stand ebenfalls auf der Tagesordnung der Konferenz, die auch die Spannung zwischen Dahomey und Niger nicht übersehen konnte. In dieser Lage ergriff Tansania die Initiative, um die OAU stärker zu engagieren und die Haltung der afrikanischen Staaten zu präzisieren. Das Ergebnis war eine Entschließung der Mitglieder der OAU, deren Präambel mit der Feststellung beginnt, daß "die Grenzen der afrikanischen Staaten, am Tage ihrer Unabhängigkeit, eine konkrete Realität darstellen". Im operativen Teil der Resolution bekräftigen die Mitgliedstaaten die strikte Achtung vor den Prinzipien nach § 3 Artikel III der Charta. Die Resolution schließt mit der feierlichen Erklärung, daß sich "alle Mitgliedstaaten verpflichten, die mit der Erlangung der nationalen Unabhängigkeit existierenden Grenzen zu achten".

Die Entschließung, die gegen den Widerstand Marokkos und Somalias mit großer Mehrheit angenommen wurde, zeigt die Hand Julius Nyereres. Er hatte bereits im Januar 1963 sein ideelles Bekenntnis zum Endziel afrikanischer Einheit mit der pragmatischen Anerkennung der kolonialen Grenzen verbunden: "Die Grenzen, die die afrikanischen Staaten teilen, machen so wenig Sinn, daß sie ohne das Bewußtsein unserer Einheit die Ursache von Spannung wären. Aber wir haben keine Alternative, als von dem Zustand auszugehen, den

Touval (oben Fn. 1), p. 85

29 Chime, Samuel, The Organization of African Unity and African Boundaries, in: Carl Gösta Widstrand (ed.), African Boundary Problems, Uppsala, 1969, p. 67. 
wir nach der kolonialen Teilung Afrikas geerbt haben. Es gibt kein Land, das nicht Gebiete einschlösse, welche unter eine andere politische Einheit fielen, wenn man Prinzipien der politischen Geographie zu Rate zöge, und zahlreiche Stämme leben wenigstens in zwei Ländern oder haben ihren Ursprung in einem anderen Teil Afrikas. Aber wenn wir jetzt gegenseitig Ansprüche auf das Gebiet des anderen erhöben, spielten wir nur in die Hände jener, die Afrika klein halten wollen... Wir müssen bei unserer Suche nach afrikanischer Einheit von der Tatsache unseres historischen Erbes ausgehen. Wir waren geteilt, so lange es Geschichte gibt."

Dabei hatte die Resolution von 1964 ihre Schwächen. Sie bot in jenen Fällen keine Hilfe, wo der Verlauf einer Grenze strittig war, die als solche nicht in Frage gestellt wurde. Sie ließ, wie im Falle Marokkos, die Frage offen, welche Bedeutung den Grenzen zukam, die schon vor der kolonialen Teilung bestanden, aber von den Kolonialmächten beseitigt worden waren. Es fällt auf, daß die Resolution nicht das Selbstbestimmungsrecht der Völker anspricht, auf das sich vor allem Somalia berief.

Stützien die "Revisionisten" ihre Ziele auf historische und ethnische Argumente, so beriefen sich die Vertreter des status quo auf die Kraft des Faktischen und das Gebot praktischer Vernunft. Ihnen war bewußt, daß ein Afrika ohne Grenzen ein wünschenswertes Ziel, aber kein realistisches Programm darstellte. Unrealistisch, aber auch gefährlich schien das Konzept einer politischen Neuordnung oder "Flurbereinigung" des Kontinents unter Anwendung ethnischer Kategorien. Gegenseitige Respektierung der bestehenden Grenzen schien das beste Mittel, die Souveränität der neuen Staaten nach außen zu sichern. Aber das koloniale Gehäuse bot auch den notwendigen Rahmen für die Konsolidierung des neuen Gebildes nach innen. Es schien dabei in Aller Interesse, den ethnischen status quo nicht zu gefährden, verkörperte er doch einen Zustand, an den man sich in kolonialen Zeiten gewöhnt hatte. Jede Änderung der Grenzen, so fürchtete man, hätte dieses Gleichgewicht gestört.

Die Unabhängigkeit hatte den neuen Staat geschaffen, aber in Afrika schafft, wie Sekou Touré es formulierte, "der Staat die Nation" ${ }^{31}$. Sie ließ sich in den ererbten kolonialen Grenzen nur selten aus einer gemeinsamen Geschichte und einem gemeinsamen ethnischen Ursprung begründen. "Nation" wurde in Afrika durch die territorialen Grenzen definiert. Bestimmte beim Augsburger Religionsfrieden von 1555 das Territorium die Religion (cujus regio, ejus religio), so in Afrika das Territorium die Nation. ${ }^{32}$ Diese Grenzen in Frage zu

Nyerere, Julius, A United States of Africa, in: Freedom and Unity, Uhuru na Umoja, Daressalam, 1966, p. 189.

31

Neuberger, Benjamin, National Self-determination in postcolonial Africa, Boulder, 1986, p. 23.

32 Zartman. William I., The Foreign and Military Politics of African Boundary Problems, in: Widstrand (oben Fn. 29), pp. 104-108; Shaw (oben Fn. 2), p. 186. 
stellen, hätte nicht nur die äußere Sicherheit, sondern auch die innere Integration der afrikanischen Staaten gefährdet, die erst im Begriff waren, auf ihrem Territorium eine Staatsnation zu erzeugen. So die Philosophie, die die Mehrzahl der afrikanischen Führer bis heute bestimmt und die Julius Nyerere schon 1963 vor norwegischen Studenten in Oslo formulierte: "Wenn das afrikanische Volk frei und in Würde leben will, muß es sich in politischen Einheiten organisieren, die groß genug sind, um die eigenen Interessen vertreten zu können... Da der Kolonialismus zur Organisation nationaler Einheiten geführt hat, müssen wir dort beginnen. Es ist wesentlich, daß wir diesen einzigen Vorteil der Zeit der Fremdherrschaft nicht verlieren: die Organisation verschiedener Stämme in einer administrativen und politischen Einheit. Dies ist so lebenswichtig, daß wir hier keine Kompromisse eingehen können. Keine Berufung auf Stammesgefühle, traditionelle Loyalitäten oder was auch immer darf die Zukunft unserer Völker gefährden." Unter Bezug auf die Ereignisse im Kongo wie den Ort seiner Rede fügte er hinzu: "Die Katangas müssen als das erkannt werden, was sie sind - die Werke afrikanischer Quislinge und ein Betrug an unserer Zukunft." 33

Die afrikanischen Führer der 60er Jahre haben ihre Option für den status quo mit ihrer eigenen Interessenlage begründet. Aber sie befanden sich dabei durchaus in Übereinstimmung mit völkerrechtlicher Theorie und Praxis. Die bei der Emanzipation Lateinamerikas von spanischer Herrschaft entwickelte Rechtsnorm des "uti possidetis" hat auch beim afrikanischen Entkolonisationsprozeß Anwendung gefunden. In ihrer historischen Form besagte sie, daß die inneren und äußeren Grenzen des spanischen Kolonialbesitzes in der Neuen Welt mit dem kritischen Datum der Unabhängigkeit dieser Gebiete von 1810 und 1821 uneingeschränkt auf die Nachfolgestaaten übergingen. Diese Norm wirkte sowohl untereinander wie auch gegen Dritte. Zwischen den Nachbarn stellte sie Klarheit her; raumfremden Staaten verweigerte sie den Zugriff. ${ }^{34}$

Der Internationale Gerichtshof in Den Haag hat in seiner Entscheidung über den Grenzstreit zwischen Burkina Faso und der Republik Mali vom 22.12.1986 die "außergewöhnliche Bedeutung" von "uti possidetis" für den afrikanischen Kontinent unterstrichen: "In diesem Zusammenhang ist festzuhalten, daß das Prinzip des uti possidetis anscheinend zuerst in Spanisch Amerika angerufen und angewandt wurde, insoweit als es dieser Kontinent war, der zuerst das Phänomen der Entkolonisierung erlebte, mit der Bildung einer Reihe souveräner Staaten auf einem Territorium, das zuvor zu einem einzigen Mutterstaat

33

34 tikk, 1963 , p. $5 / 6$.

Shaw (oben Fn. 2), pp. 187/188; Torres Bernárdez, Santiago, The "Uti Posseditis Juris Principle" in Historical Perspective, in: Konrad Ginter u.a. (Hrsg.), Völkerrecht zwischen normativem Anspruch und politischer Realität, Festschrift für Karl Zemanek, Berlin, 1994, S. 417-437; abweichend McEwen (oben Fn. 23), p. 27-31. 
gehörte. Nichtsdestoweniger ist das Prinzip nicht eine spezielle Regel, die sich nur auf ein spezifisches System des Völkerrechts bezieht. Es ist ein allgemeines Prinzip, das logisch mit der Erlangung der Unabhängigkeit verbunden ist, wo immer sie stattfindet. Sein offensichtlicher Zweck ist es, zu verhindern, daß die Unabhängigkeit und Stabilität neuer Staaten durch Bruderstreit gefährdet wird, der durch die Infragestellung von Grenzen nach dem Rückzug der bisher verwaltenden Macht provoziert würde." Zusammenfassend stellte der Internationale Gerichtshof fest, daß es sich hier nicht um die allmähliche Entwicklung eines Prinzips des Völkergewohnheitsrechts handle, das sich nur auf Afrika wie früher auf Spanisch Amerika beziehe, sondern um eine auf Afrika angewandte Regel von allgemeiner Bedeutung. ${ }^{35}$

Die Respektierung der kolonialen Grenzen, wie sie von den Afrikanern praktiziert und im uti possidetis formuliert wurde, ist eng mit der Frage der Staatennachfolge verbunden. Die VN-Konferenz über die Staatennachfolge in Verträgen hat mit ihrer Konvention vom 22.8.1978 den im Zuge der Entkolonialisierung neu entstandenen Staaten im Hinblick auf die Verträge des Vorgängerstaates ein großes Maß an Entscheidungsfreiheit eingeräumt und ihnen damit die Möglichkeit eines neuen Anfangs (clean slate) gegeben. Anders ist es bei Verträgen mit territorialem Bezug, wo es von geringerer Bedeutung ist, wer die Verträge abgeschlossen hat. Der Rechtszustand, den sie begründen, "haftet ... an dem Gebiet, und die daraus folgenden Rechte und Pflichten sind solche des Staates, der jeweils das Gebiet in Besitz hat" ${ }^{36}$. Anders ausgedrückt: Das Territorium und seine Grenzen stehen nicht zur Disposition. Dies ist die Konsequenz der Staatennachfolge, aber auch ihre Voraussetzung. Es sei denn, alle von der Staatennachfolge Betroffenen würden einvernehmlich anderes beschließen.

\section{Die Respektierung der kolonialen Grenzen: Das Dilemma}

Der Sezessionsversuch Katangas (1960-1963) hat dazu beigetragen, die Respektierung der kolonialen Grenzen in der Charta der OAU zu verankern. Aber die Beschlüsse der OAU von 1963 und 1964 haben nicht verhindert, daß die überwiegend von den Ibo bewohnte östliche Provinz Nigerias sich am 30.5.1967 als "Biafra" unabhängig erklärte. Obwohl Nigeria die Sezession Biafras als seine innere Angelegenheit betrachtete, haben sich OAU und afrikanische Staatsmänner bemüht, die beiden Bürgerkriegsparteien zu einer friedlichen Beilegung ihres Streits zu bewegen. Dabei hat die Sonderkonferenz der OAU in Kinshasa vom September 1967 an ihrer grundsätzlichen Haltung keinen Zweifel gelassen. Sie verurteilte jede Sezession in einem Mitgliedsstaat und beauftragte eine Delegation von 
sechs Staatsoberhäuptern, den nigerianischen Staatschef ihrer grundsätzlichen Unterstützung zu versichern. Am Ende ihrer Mission erklärten die Staatsoberhäupter, daß eine Lösung der Krise nur auf der Grundlage der Einheit Nigerias und seiner territorialen Integrität gefunden werden könne. ${ }^{37}$ Im November 1967 bekräftigte Kaiser Haile Selassie von Äthiopien einen allgemeinen Konsens der OAU, wonach "die nationale Einheit und die territoriale Integrität der Mitgliedsstaaten nicht verhandelbar" seien. ${ }^{38}$

In dieser Position zeigten sich ernstliche Risse, als im April und Mai 1968 zuerst Tansania, dann Gabun, die Elfenbeinküste und Sambia Biafra als souveränen Staat anerkannten. Diese Kehrtwendung erfolgte vor allem unter dem Eindruck des Krieges gegen die Ibo, der von zahlreichen Staaten als Massaker und Pogrom empfunden wurde. Tansanias Präsident Nyerere begründete seine Haltung damit, "daß wir, indem wir die Existenz Biafras nicht anerkannten, schweigend einen Krieg gegen das Volk von Ostnigeria unterstützten - einen Krieg im Namen der Einheit... Aber Einheit kann nur auf die allgemeine Zustimmung des betroffenen Volkes gegründet sein. Das Volk muß fühlen, daß dieser Staat der seine ist... Denn Staaten und Regierungen existieren für den Menschen und den Dienst a m Menschen. Sie sind da für den Schutz ihrer Bürger, ihre Wohlfahrt und die künftige Wohlfahrt ihrer Kinder. Es gibt für Staaten und Regierungen keine andere Rechtfertigung als den Menschen." Er sei, so Nyerere, im Grunde gegen eine Sezession. Als Präsident von Tansania habe er geschworen, die Integrität der Vereinigten Republik zu bewahren: "Aber wenn die Masse der Bevölkerung von Sansibar sich ohne äußere Manipulation aus eigenen Gründen entscheiden sollte, daß die Union für ihre Existenz nachteilig sei, würde ich nicht dafür sein, sie in die Unterwerfung zu bomben... Ich könnte keinen Krieg gegen das Volk führen, dem zu dienen ich geschworen habe... Wir glauben in diesem Land, daß die Einheit für die Zukunft Afrikas lebenswichtig ist. Aber es muß eine Einheit sein, die dem Volke dient und die frei vom Volk bestimmt wird." ${ }^{39}$ Auf der Sechsten Versammlung der OAU im September 1969 wurde bei Stimmenthaltung von fünf Staaten eine Resolution verabschiedet, die beide Seiten aufforderte, im vorrangigen Interesse Afrikas die Einheit Nigerias zu bewahren. Am 21.1.1970 kapitulierte Biafra.

Mit großer Mehrheit haben die afrikanischen Staaten das von Biafra angerufene und von Nyerere unterstützte Selbstbestimmungsrecht der Völker als nicht anwendbar erklärt und das Prinzip der territorialen Integrität bekräftigt. Für die Mehrheit der afrikanischen Staaten blieben die kolonialen Grenzen sakrosankt. Aber damit waren Afrikas territoriale Probleme nicht gelöst. Ungeklärt blieben auch die Fragen, die sich durch das Spannungsverhältnis 
von Völkerrechtsnormen ergaben, auf die sich die Mitglieder der OAU selektiv bezogen. ${ }^{40}$ Hier sind neben dem Souveränitätsanspruch der Staaten das Selbstbestimmungsrecht der Völker, das Einmischungsverbot, der Gewaltverzicht und das Prinzip der territorialen Integrität betroffen. Ihr dialektisches Verhältnis ist auch für das Problem der kolonialen Grenzen von Bedeutung.

Von einer moralisch-politischen Forderung hat sich das Selbstbestimmungsrecht der Völker im Laufe dieses Jahrhunderts zu einer universellen Norm des Völkerrechts entwickelt. ${ }^{41}$ Unbestritten war, daß die Dekolonisierung eine Konkretisierung des Selbstbestimmungsrechts darstellte, wobei den Erklärungen der Generalversammlung der VN vom 14.12.1960 und 24.10.1970 besondere Bedeutung zukommt. ${ }^{42}$ Die Diskussion dieser Jahre zeigt die Tendenz der Dritten Welt, die Anwendung des Prinzips der Selbstbestimmung auf die kolonialen Staaten zu beschränken. Sie zeigt zugleich den instrumentalen Charakter dieses Prinzips, das als Mittel zur Durchsetzung der Unabhängigkeit verstanden wird. Das Selbstbestimmungsrecht kann sich dabei im Zeitpunkt der Unabhängigkeit in der Errichtung eines eigenen souveränen Staates - in den kolonialen Grenzen - wie in der staatlichen Verbindung mehrerer Territorien - in deren Grenzen - äußern. Auch eine spätere Integration unabhängiger Staaten zu einem Bundesstaat - wie 1964 im Falle der Union von Sansibar und Tanganyika - bleibt möglich. Sonst aber gilt das Selbstbestimmungsrecht als konsumiert. Es läßt sich nicht gegen einen widerstrebenden Nachbarn - zum Zwecke einer Grenzkorrektur - verwenden. Es bietet den Minderheiten im eigenen Land kein Mittel der Berufung. Es schließt das Recht auf Sezession aus. So das Verständnis der Mehrheit der afrikanischen Staaten. So auch das Verständnis der Vereinten Nationen jener Jahre, deren Generalsekretär U Thant am 9.1.1970 in Dakar im Zusammenhang mit Biafra erklärte: "Was die Frage der Abtrennung eines bestimmten Gebiets eines Mitgliedstaates betrifft, so ist die Haltung der Vereinten Nationen unmißverständlich. Als internationale Organisation haben die Vereinten Nationen niemals das Prinzip der Sezession eines Teils eines Mitgliedstaates akzeptiert. Sie akzeptieren es nicht, und ich glaube, sie werden es nie akzeptieren. ${ }^{43}$

Rumpf, Helmut, UNO-Prinzipien im Widerspruch, in: Ingo v. Münch (Hrsg.), Festschrift für HansJürgen Schlochauer, Berlin, 1981, S. 576-602.

41

Hierzu Partsch, Karl Josef, Selbstbestimmung, in: Rüdiger Wolfrum (Hrsg.), Handbuch Vereinte Nationen, München, 1991, S. 745-752; Doehring. Karl, Das Selbstbestimmungsrecht der Völker, in: Bruno Simma (Hrsg.), Charta der Vereinten Nationen, München, 1991, S. 15-32.

42

Tomuschat, Christian, Self-Determination in a Post-colonial World, in: Christian Tomuschat (ed.), Modem Law of Self-Determination, Dordrecht, 1993, pp. 1-20.

43

Buchheit, Lee C., Secession. The Legitimacy of Self-Determination, New Haven, 1978, p. 87. 


\section{Prinzipien im Konflik: Sezession als Ausweg?}

Trotz der dezidierten Worte des damaligen Generalsekretärs der Vereinten Nationen hat sich seit den 60er Jahren im Afrika südlich der Sahara in einer Reihe von Fällen die Frage der kolonialen Grenzen in der Form der Sezession gestellt. Die Fälle Katanga und Biafra sind dabei als gescheiterte Versuche in die Geschichte eingegangen. Eritrea ist es dagegen gelungen, seine Selbständigkeit gegen Äthiopien zu erkämpfen. Ende des 19. Jahrhunderts als italienische Kolonie entstanden, wurde Eritrea nach dem Zweiten Weltkrieg durch Beschluß der Vereinten Nationen mit Äthiopien in einer Föderation vereint, bis Haile Selassie 1962 Eritrea als 14. Provinz annektierte. Ab 1961 begann der bewaffnete Kampf, der sich seit 1970 erheblich verschärfte, wobei eine Reihe islamischer wie nichtislamischer afrikanischer Staaten die eritreischen "Separatisten", die Sowjetunion und Kuba Äthiopien unterstützten. $^{44}$ Als sich 1977 der Sudan offen zu den Eritreern bekannte, beschuldigte Äthiopien den Sudan, die Charta der OAU und die territoriale Integrität Äthiopiens zu verletzen, die für die Eritreer auf der Kolonialherrschaft eines schwarzafrikanischen Landes über seinen Nachbarn beruhte. Was den Äthiopiern als Sezession erschien, war für die Eritreer die (Wieder-) Herstellung ihrer Souveränität auf der Grundlage des Selbstbestimmungsrechts der Völker. ${ }^{45}$ Nach mehr als 10 Jahren kriegerischer Auseinandersetzung hat erst der militärische Kollaps des Mengistu-Regimes Eritrea den Weg zur Unabhängigkeit geebnet. Das unter der Überwachung der Vereinten Nationen durchgeführte Referendum hat 1993 diese Unabhängigkeit mit über $99 \%$ der Stimmen legitimiert. Zwei Monate später wurde Eritrea in die Organisation für afrikanische Einheit aufgenommien, die es die ganze Zeit vermieden hatte, in diesem Konflikt Stellung zu beziehen. ${ }^{46}$

Dagegen ist eine Ende des Bürgerkrieges im Sudan nicht abzusehen, der bereits ein Jahr vor der Unabhängigkeit (1956) einsetzte. Die Vertreter der Regierung in Khartum verweisen darauf, daß der unter britischer Herrschaft vereinte Sudan das Recht der Selbstbestimmung gemeinsam ausgeübt habe und dieser Schritt unumkehrbar sei. Der Süden hat demgegenüber die Auffassung vertreten, daß eine echte Verbindung beider Gebiete nie bestanden habe und der Süden praktisch eine Kolonie des Nordens gewesen sei, der auch das Recht der Selbstbestimmung usurpiert und die Zusage einer föderativen Staatsstruktur nicht eingehalten habe. Sehen sich die Nordsudanesen als Teil einer moslemisch-arabischen, so die Südsudanesen als Teil einer schwarzafrikanischen Kultur. ${ }^{47}$ Dieser Gegensatz hat sich bis heute als unüberbrückbar erwiesen. Ein politisches System, das auf dem islamischen

44 45

46

47

Neben Nordafrikanischen arabischen Staaten ab 1981 auch die Elfenbeinküste, Togo, Benin, Niger, Somalia, Malagassische Republik, Mosambik. Siehe Neuberger (oben Fn. 31), S. 80.

Gayim, Eyassu, The Eritrean Question, Uppsala, 1993, p. 577; siehe auch Matthies, Volker, Äthiopien, Eritrea, Somalia, Djibouti, München, 1997.

United Nations, The United Nations and the Independence of Eritrea, New York, 1996.

Neuberger (oben Fn. 31), p. 38. 
Recht der Scharia beruhe, sei für die Bewohner des Südens nicht akzeptabel, so Rebellenführer John Garang Ende 1997 in Kairo: "Wir sind nicht gegen den Islam, doch Religion muß vom Staat getrennt werden." Auch sei man nicht gegen die sudanesische Einheit, aber "wir sind für eine Einheit, die allen Sudanesen gleiche Rechte gibt". Ob Garang und seine Anhänger sich mit dem Ziel eines letztlich säkularen Staates gegenüber einem fundamentalistischen Regime durchsetzen können, wird den meisten als fraglich erscheinen. ${ }^{48}$

Aber der Bürgerkrieg ist nicht nur für die Betroffenen von existentieller Bedeutung; er berührt auch die Staatsraison der afrikanischen Staaten, die offiziell auf der Respektierung der kolonialen Grenzen beruht. Dennoch hat der Süden in seiner Auseinandersetzung die Unterstützung Äthiopiens, Zaires, Ugandas und Eritreas gefunden, auch wenn diese Staaten - auch aus Furcht vor Repressalien - gezögert haben, sich offen zur Sezession des Südens zu bekennen und sich damit in Gegensatz zum Nichteinmischungsgebot der OAU zu setzen. $^{49}$ Die Verbindung der Rebellenbewegung des Südens (SPLA) mit Oppositionskräften des Nordens hat die Lage weiter kompliziert. Um so weniger ist von der OAU ein Beitrag zur Lösung der Probleme zu erwarten.

Ungelöst wie der Fall Sudan ist auch die somalische Frage. Sie ist älter als die OAU, mit deren Existenz sie eng verbunden ist. Schon auf der Gründungskonferenz von 1963 hatte der somalische Präsident Aden Abdullah Osman das Selbstbestimmungsrecht für die der somalischen Republik benachbarten Gebiete gefordert. Es war daher konsequent, daß sich Somalia den Beschlüssen von 1964 widersetzte, da sie dem somalischen Ziel der Revision seiner Grenzen widersprachen. Ziel des somalischen "Irredentismus" war die "Befreiung der somalischen Territorien von kolonialer Bedrückung", wie es die Verfassung von 1973 formulierte. ${ }^{50}$ Als eines der wenigen ethnisch homogenen Länder Afrikas war Somalias Politik darauf gerichtet, die "amputierten" Glieder seines Volkskörpers zu einem "Größeren Somalia" zu verbinden. Davon waren die von Somalis bewohnten Gebiete Äthiopiens, Kenias und Dschibutis betroffen. Die fünf Sterne der somalischen Flagge erinnern an dieses Ziel.

Der Rückblick auf die Geschichte enthüllt eine komplexe Situation, die mit der OAU-Formel von 1964 schwer zu erfassen ist. Während die OAU den Staat durch sein Territorium in den kolonialen Grenzen definierte, verfügte Somalia als ethnisch begründeter "Nationalstaat" über eine raison d'être, die keiner Legitimation durch koloniale Grenzen bedurfte. Nicht akzeptabel aber war für die Somali, daß die Grenzen, wie sie zum Zeitpunkt der Unabhängigkeit bestanden, die Somali außerhalb dieser Grenzen unter fremde Herrschaft

48

49

50
Frankfurter Allgemeine Zeitung, 9.12.1997, S. 9.

Shaw (oben Fn. 2), p. 211; Touval (oben Fn. 1), p. 154; Ansprenger, Franz, Politische Geschichte Afrikas im 20. Jahrhundert, München, 1997, S. 162.

Neuberger (oben Fn. 31), p. 86. 
stellten. Kompliziert wurde die Lage durch die Zahl der betroffenen Parteien und die Verbindung kolonialer Grenzziehung (durch Großbritannien, Italien und Frankreich) mit der eines afrikanischen Staates: Äthiopien.

Der britische Außenminister Ernest Bevin hatte 1946 dem Unterhaus einen Vorschlag zur Lösung der somalischen Frage unterbreitet, als sich nach dem zweiten Weltkrieg die Möglichk :it bot, die Grenzen zu revidieren, die seit der zweiten Hälfte des vergangenen Jahrhunderts das Horn Afrikas teilten: "Zu der Zeit, als wir unseren Teil besetzten, besetzten die Äthiopier ein Gebiet im Inneren, das für die Hälfte der Nomaden von Britisch-Somaliland sechs Monate im Jahr die Weidefläche dar ' 'llt. Auf ähnliche Weise müssen auch die Nomaden von Italienisch-Somaliland auf der Suche nach Gras die bestehenden Grenzen überqueren. Wir haben daher, mit den besten Absichten, vorgeschlagen, daß Britisch- und Italienisch-Somaliland mit dem angrenzenden Teil Äthiopiens, sofern Äthiopien zustimmt, als Treuhandgebiet vereinigt werden sollte, so daß die Nomaden ihre bescheidene Existenz mit der geringsten Behinderung fristen könnten und es für sk eine wirkliche wirtschaftliche Chance gäbe, so wie man sie in dieser Region versteht. ${ }^{51}$

Dieser Vorschlag Bevins setzte sich nicht durch. 1948 übergab Großbritannien Ogaden, 1954 Haud und "die reservierten Gebiete" wieder den Äthiopiern. 1960 wurde das aus dem britischen und italienischen Teil des Landes vereinigte Somalia unabhängig. Seitdem kämpfte es um die Angliederung jener äthiopischen Gebiete an Somalia, die von Somali bewohnt sind. Ziel Somalias war aber auch der Anschluß des kenianischen Northern Frontier District (NFD), der über eine überwiegend somalische Bevölkerung verfügt. Kenia wie Äthiopien haben ihre Weigerung, mit Somalia in Verhandlungen einzutreten, mit dem Argument begründet, daß das Prinzip der Selbstbestimmung auf unabhängige Staaten in Afrika keine Anwendung finde. Das einzige Selbstbestimmungsrecht, das die Somali beanspruchen könnten, sei das nomadische: "ihre Kamele zu beladen und nach Somalia zu ziehen", wie es Jomo Kenyatta formulierte ${ }^{52}$. Die äthiopischen Argumente unterschieden sich dabei in der revolutionären Ära Mengistu Haile Mariams nicht von denen Haile Selassies, die beide den Domino-Effekt der somalischen Irredenta und der eritreischen Abspaltung fürchteten - mit dem Ergebnis einer Reduktion Äthiopiens auf einen vom Meer abgeschnittenen amharischen Kern. Die Somali haben dem entgegengehalten, daß der äthiopische Staat durch die Ethnie der Amhara bestimmt sei, für die alle anderen auf dem Staatsgebiet ansässigen Ethnien nur koloniale Subjekte darstellten. Für die Somali ist Äthiopien der letzte Kolonialstaat auf afrikanischem Boden, der sich weigert, den von ihm beherrschten Völkern das Selbstbestimmungsrecht einzuräumen und dem es daher nicht gestattet sei, 
sich auf die Beschlüsse der OAU von 1964 zu berufen. ${ }^{53}$ Angesichts der "selbstinszenierten Zerstörung eines Staatswesens" ${ }^{54}$ waren in den letzten Jahren jedoch nicht die äußeren Grenzen Somalias, sondern die innere Spaltung das eigentliche Problem. Mit der Ausrufung der sogenannten "Republik Somaliland" im Mai 1991 stellte sich für manche die Frage, ob in Afrika das Modell des ethnisch einheitlichen "Nationalstaats." dem Modell der multiethnischen "Staatsnation" a priori überlegen sei. In der Sicht der "Republik Somaliland", die im wesentlichen der früheren britischen Kolonie gleichen Namens entspricht, handelte es sich bei der Abspaltung vom Gesamtstaat nicht um den Fall einer Sezession, sondern die legale Auflösung der Union von 1960. Ebenso wenig bedeute die Unabhängigkeit einen Verstoß gegen die OAU-Norm von 1964, "da man ja keine Kolonialgrenzen verändere, sondern sie ganz im Gegenteil wieder herstelle" ${ }^{\text {"55 }}$. Es bleibt abzuwarten, ob mit der Ende 1997 in Kairo zwischen 26 verfeindeten Gruppen beschlossenen "Konferenz zur nationalen Versöhnung" der Bürgerkrieg in Somalia sein Ende findet.

\section{Prinzipien im Konflikt: Territoriale Integrität und Selbstbestimmungsrecht}

So unterschiedlich die Fälle Eritrea, Sudan und Somalia zu bewerten sind, zeigen sie doch, daß die OAU-Formel von der Respektierung der kolonialen Grenzen dort nicht akzeptiert wird, wo für die Betroffenen Interessen auf dem Spiel stehen, die sie als existentieller betrachten als die Beachtung einer rechtlichen Norm. Im Spannungsfeld von Selbstbestimmung und territorialer Integrität geht es dabei um das "Recht" auf Sezession.

Völkerrecht wie Staatenpraxis sind dabei grundsätzlich sezessionsfeindlich. Das Souveränitätsrecht der Staaten wie die Prinzipien der Nichteinmischung und der territorialen Integrität stehen der Sezession im Wege. In ihrer "Erklärung über die Entkolonisierung" von 1960 hat die Generalversammlung der Vereinten Nationen entsprechend festgestellt, "daß jeder Versuch, der sich auf die teilweise oder völlige Auflösung der nationalen Einheit und der territorialen Integrität eines Landes richtet, mit den Zwecken und Prinzipien der Charta der Vereinten Nationen nicht vereinbar ist" ${ }^{56}$. Die Vereinten Nationen haben ihren Standpunkt und ihre Ablehnung der Sezession in ihrer grundlegenden Erklärung über den Verkehr zwischen den Staaten von 1970 bekräftigt. Sie haben ihre allgemeine Ablehnung der Sezession allerdings vorsichtig dadurch beschränkt, daß sie den Schutz der territorialen Integrität und der nationalen Einheit der Staaten davon abhängig machen, daß diese Staaten "über eine Regierung verfügen, die das gesamte zum Gebiet gehörige Volk ohne Unter-

Neuberger (oben Fn. 31), pp. 84-91; Matthies, Volker, Der Grenzkonflikt Somalias mit Äthiopien und Kenya, Hamburg, 1977; Krech, Hans, Der Bürgerkrieg in Somalia (1988-1996), Berlin, 1996.

Tetzlaff, Rainer : Staatszerfall und staatliche Neugliederung, in: Afrika-Jahrbuch 1992, S. 26. 
schied der Rasse, des Glaubens oder der Hautfarbe vertritt". Nur der Staat, der das Recht der Selbstbestimmung achtet, verdient den Schutz seiner territorialen Integrität. ${ }^{57}$ So die Interpretation und Argumentation, die in den letzten Jahren an Boden gewinnt. Das Selbstbestimmungsrecht der Völker erscheint dabei als eine Norm, die die Rechte der Menschen einschließt, die diese Völker bilden.

Dabei sind sich die Vertreter dieser Tendenz der Gefahren bewußt, die sich aus einem Recht auf Sezession ergeben. Ist schon, wie es US-Präsident Woodrow Wilsons Außenminister Robert Lansing 1919 formulierte, die Forderung nach nationaler Selbstbestimmung Dynamit, so wird vielen Politikern ein Recht auf Sezession als potenziertes Instrument der Zerstörung der internationalen Ordnung erscheinen. Aber es ist gerade der Schutz dieser internationalen Ordnung, die nach Meinung anderer das Recht auf Sezession als ultima ratio erfordert. Sie stimmen darin überein, daß, solange in einem Vielvölkerstaat Menschen- und Minderheitenrechte, d.h. die "innere" Selbstbestimmung, beachtet werden, das Recht auf Selbstbestimmung nicht in ein Recht auf Sezession münden kann. Ist dies jedoch nicht der Fall, dann ist für Kimminich das Recht auf Sezession "eine Konsequenz der Verweigerung des Selbstbestimmungsrechts. Die Ströme von Blut, die Senator Lansing vorausgesehen hat, sind nicht wegen der Verwirklichung, sondern wegen der Verweigerung des Selbstbestimmungsrechts geflossen" ${ }^{\prime 58}$. Tomuschat argumentiert ähnlich, wenn er Bevölkerungsgruppen von der Loyalitätspflicht gegen einen Staat entbindet, der sie mit seinem Terrorapparat bedroht. ${ }^{59}$ Für Murswiek wäre ohne das Sezessionsrecht im Falle unmäßiger, auf anderem Wege nicht zu beseitigender Diskriminierung das Selbstbestimmungsrecht nichts als ein "leeres Gehäuse" ${ }^{60}$. Bleibt auch für die genannten Völkerrechtler die Sezession eine latente und in extremis legitime Option, so scheint es ihnen angesichts der Konsequenzen einer Sezession opportuner, das Selbstbestimmungsrecht durch Minderheitenschutz, innere Autonomie oder eine föderative Staatsstruktur zu verwirklichen: "Autonomie ist der beste Schutz vor Sezession, wenn rechtzeitig gewährt. Wer zu spät kommt, ... wird von der Geschichte bestraft." 61

Das Völkerrecht hat jedoch bis heute den akademischen Diskurs nicht rezipiert und verharrt auf dem Stand der VN-Erklärung von 1970. Dabei ist das letzte Vierteljahrhundert von

57 
einer Serie von Sezessionen bestimmt, bei der Bangladesh 1970 den Auftakt bildete. Staaten sind nicht mehr unteilbar, wie die Auflösung der Sowjetunion, die W'iederherstellung der baltischen Staaten und der Zerfall Jugoslawiens beweisen. Tschetschemen und Bosnien - unterschiedlich, wie die Fälle sind - zeigen zugleich die Problematik dieser Prozesse.

Hat in Europa die Sezession ihren Tabucharakter verloren, so bleibt für das afrikanische Establishment die Respektierung der kolonialen Grenzen oberstes Gebot. Aber auch in Afrika besteht eine Kluft zwischen Theorie und Praxis. Es stellt sich die Frage, welche Konsequenzen sich daraus ergeben.

\section{Von der äußeren zur inneren Selbstbestimmung}

Es steht außer Zweifel, daß die Beschlüsse der OAU von 1963 und 1964 insgesamt die afrikanischen Grenzen stabilisiert und die afrikanischen Staaten in diesen Grenzen konsolidiert haben. Dies ist keine geringe Leistung, wenn man den kolonialen Ursprung dieser Grenzen berücksichtigt, die heute 53 Staaten des Kontinents voneinander trennen. Zugleich hat sich in den meisten Staaten eine nachkoloniale Identität entwickelt, die in den Worten Léopold Senghors die vorkolonialen Stammeseinheiten "transzendiert". Der Internationale Gerichtshof konnte daher 1986 im Streit zwischen Burkina Faso und der Republik Mali feststellen, daß man "die Erhaltung des territorialen status quo in Afrika als den klügsten Weg betrachtet, um das zu bewahren, was von den Völkern erreicht wurde, die für ihre Unabhängigkeit kämpften und um eine Aufspaltung zu vermeiden, die dem Kontinent jene Vorteile nehmen würde, die man mit so vielen Opfern erreicht hat. Das wesentliche Erfordernis der Stabilität, die notwendig ist, um zu überleben, um sich zu entwickeln und allmählich ihre Unabhängigkeit auf allen Gebieten zu festigen, hat die afrikanischen Staaten veranlaßt, voller Einsicht der Respektierung der kolonialen Grenzen zuzustimmen und diesem Gesichtspunkt in der Interpretation des Prinzips des Selbstbestimmungsrechts der Völker Rechnung zu tragen" 62 . Indem der Internationale Gerichtshof in abwägender Sprache einen (weitgehenden) afrikanischen Konsens registriert, deutet er zugleich an, daß in der Doktrin und Praxis der OAU das Prinzip der territorialen Integrität das Prinzip der Selbstbestimmung relativiert. Uns stellt sich die Frage, ob die Beschlüsse der OAU von 1963 und 1964 in heutiger Sicht nicht zu sehr auf den status quo fixiert sind.

Denn die Beschlüsse von einst schreiben politische Positionen und Prioritäten fest, die nicht in vollem Umfang die Entwicklung Afrikas und des Völkerrechts berücksichtigen. Gefordert sind heute weniger feste Doktrinen als flexible Reaktionen, die einer of t komplexen Lage gerecht werden. Weder garantiert der territoriale status quo den Frieden Afrikas noch löst seine Beseitigung Afrikas Probleme. Sowohl Bewahrung wie Veränderung haben

62

I.C.J. Reports 1986, p. 17. 
ihren Wert. Afrika braucht eine Grundnorm wie die Schlußakte der KSZE, die der "Unver1.: :Llichkeit" (nicht "Unveränderlichkeit") der Grenzen ebenso Rechnung trägt wie der Möglichkeit des "friedlichen Wandels". Statt der "Heiligen Allianz" in Addis Abeba braucht Afrika seine "Organisation für Sicherheit und Zusammenarbeit" (OSZA), die anders als die OAU nicht durch Einmischungs- und Sanktionsverbote gelähmt wird. Dies erscheint um so notwendiger, als der Westen und die Vereinten Nationen immer stärker zögern, sich in Afrika politisch oder militärisch zu engagieren.

Eine Generation nach Erlangung der Unabhängigkeit geht es nicht so sehr um die Festigung der Souveränität nach außen wie um die Festigung der Demokratie nach innen. Nicht die Grenzen sind heute Afrikas wichtigstes Problem, sondern der Zustand innerhalb der Grenzen. Hier sind die Mitglieder der OAU in der Pflicht, die von ihnen ratifizierte "Afrikanische Charta über die Rechte der Menschen und Völker" von 1981 mit Leben zu erfüllen. ${ }^{63}$ Dies wäre nicht nur ein Beitrag zur Sicherung der individuellen Rechte des afrikanischen Bürgers, sondern zugleich - im Eigeninteresse seiner Regierungen - ein wesentlicher Beitrag zum inneren Ausgleich und damit zur Akzeptanz des Staates und seiner Grenzen. Die "Öffnungen" des nationalstaatlichen Konzepts, die Hans Maier für Ost- und Südosteuropa gefordert hat, sind für Afrika von gleicher Bedeutung: Minderheitenschutz und Minderheitenvertretung; individuelle, soziale und kulturelle Menschenrechte; eine föderalistische Staatsorganisation, die das Eigenleben von Regionen und Minderheiten stärkt; Bereitschaft zur internationalen Zusammenarbeit und zur übernationalen Integration. ${ }^{64}$

In diesem Konzept verlieren auch die Grenzen an Bedeutung. Afrikas Entwicklung, seine Wohlfahrt und seine Stabilität erfordern politische und wirtschaftliche Zusammenarbeit in regionalem und kontinentalem Rahmen. Der Nationalstaat war die angemessene Antwort auf die Probleme von gestern. Auch heute erfüllt er noch seinen Zweck. Aber er ist wohl nicht die letzte Antwort auf die Probleme von morgen. Bis dahin ist es in Afrika noch ein langer Weg. Aber erst, wenn die Grenzen ihre heutige Bedeutung verlören, würde Afrika jene Teilung überwinden, für die die Berliner Konferenz von 1884/85 zum Symbol geworden ist. Protection of Human Rights by International Law: The Emerging African System, Baden-Baden, 1985. Kritisch zu Defiziten der Charta: Kühnhardt, Ludger, Stufen der Souveränität. Staatsverständnis und Selbstbestimmung in der "Dritten Welt", Bonn, 1992.

Frankfurter Allgemeine Zeitung, 13.11.1997, S. 43. 


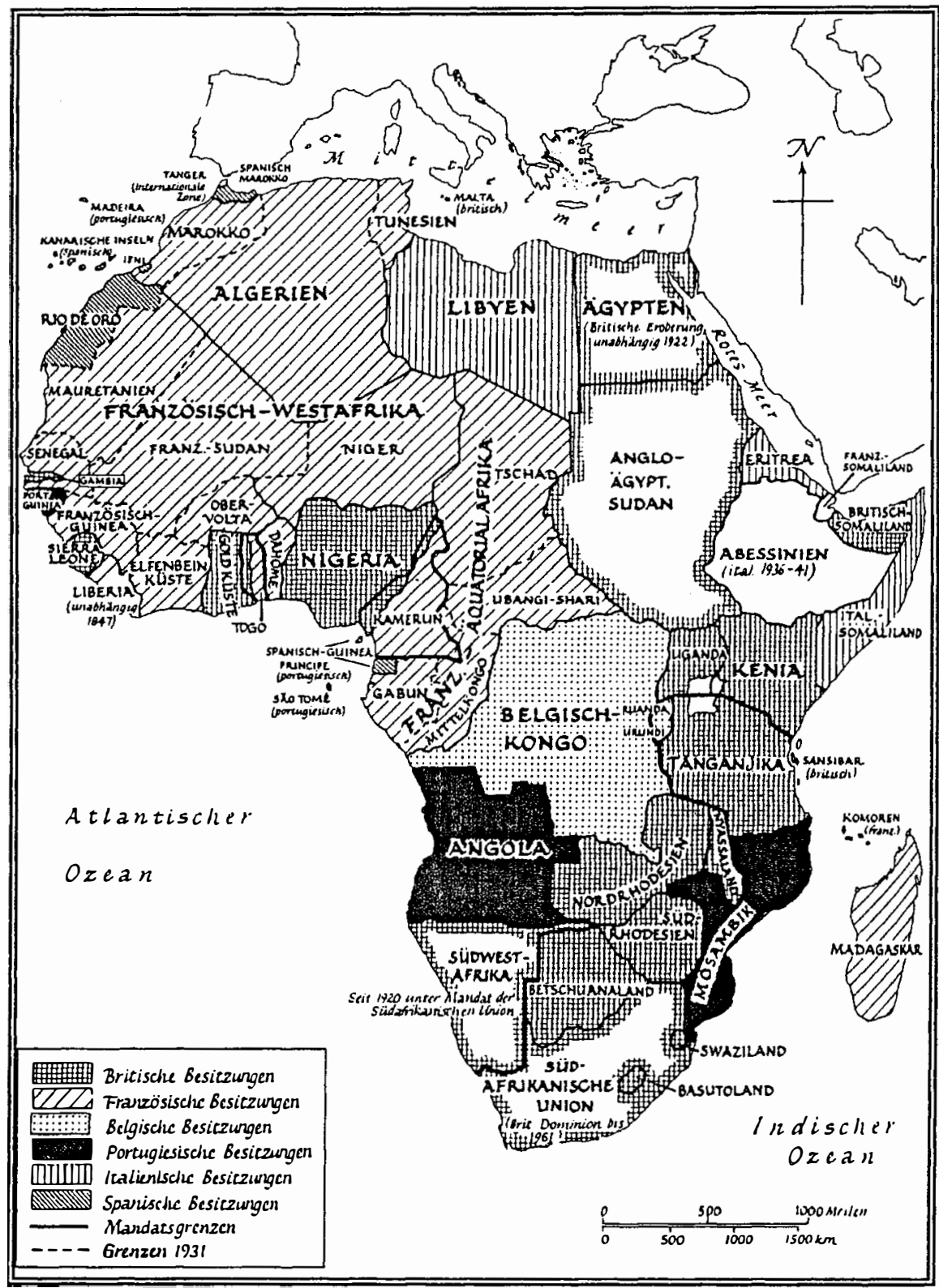

$A b b .1$ : Kolonialgrenzen

Quelle: Roland Oliver, The African experience, London 1991 


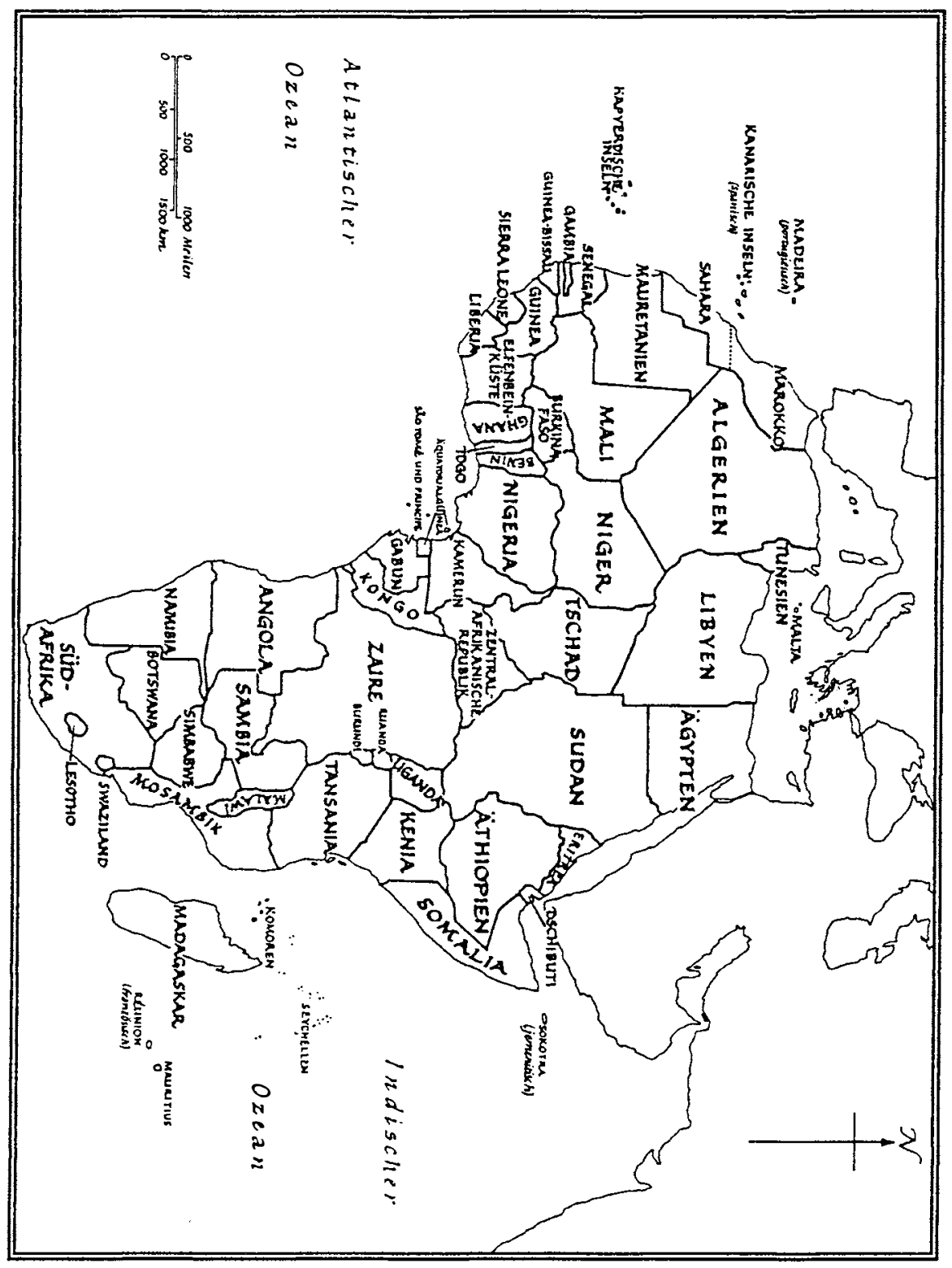

$A b b$. 2: Die Staaten des unabhängigen Afrika

Quelle: Roland Oliver, The African experience, London 1991 
Africa's "artificial" boundaries: A colonial burden or a sensible solution?

\section{By Heinz Schneppen}

The boundaries established in colonial times are still the boundaries of most African states. Before independence, African leaders asked for a revision of these boundaries, which they considered arbitrary and artificial. When independence came, African leaders had changed their minds. A majority of African states opted for the preservation of the status quo, in agreement with international practice. A "revisionist" minority however ignored and challenged the doctrine of the OAU (which is to respect the boundaries as they were when the States achieved independence.)

There can be no doubt that the OAU-doctrine has contributed to the stabilization of states and boundaries in Africa. But events in Biafra, Eritrea, Sudan and Somalia have demonstrated that the principle of territorial integrity can conflict with the principle of selfdetermination (which is at least of equal importance).

In this context, secession might be considered a means of last resort, when selfdetermination is denied. But given the sometimes disastrous results of secession, states in Africa would be well advised to forestall the possibility of secession by granting internal self-determination (minority rights and autonomy) in time. The OAU would do well to abandon its rigid stance in favor of a more flexible position. African states should transform their organization, up to now lacking sanctions as well as resources, into an efficient (Organization for Security and Cooperation in Africa" (OSCA). Boundaries, in addition, would lose much of their devisive (and explosive) character through continental cooperation and regional integration. If African states could proceed in this way, the question as to whether their boundaries are artificial or reasonable would become rather academic.

The cameroonian land law in view of the constitutional regulations to protect the rights of autochtonous populations

\section{By Prosper Nkou Mvondo}

The new cameroonian Constitution of January 18, 1996, poses the principle of "protection of the rights of autochtonous populations". The autochtonous is defined in relation to land. The first right of an autochtonous population is then first of all, a right on his land of birth. By posing the principle, it stands to reason that the constitution calls for a land reform which will integrate the notion of autochtonous population, well known in the sociological domain, but ignored up to now by the cameroonian legal order in general and by the land 